\title{
DESENVOLVIMENTO DE JOGO ELETRÔNICO COMO INTERVENÇÃO DE EDUCAÇÃO EM SAÚDE PARA O ADOLESCENTE
}

\author{
Rosa Hiolanda Abreu de Sousa, Universidade Federal do Pará, rosahiolanda22@gmail.com \\ Abner Cardoso da Silva, Universidade Federal do Pará, abner.cardo@gmail.com \\ Daniel Felipe Lopes de Deus, Universidade Federal do Pará, danielfdeus@ gmail.com \\ John Lucas Rodrigues Portilho de Sousa, Universidade Federal do Pará, kresteagle@ gmail.com \\ Gilberto Nerino de Souza Junior, Universidade Federal do Pará, gilbertonerinojr@gmail.com \\ Liliane Silva do Nascimento, Universidade Federal do Pará, lilianenascimento2001 @ gmail.com
}

Resumo. A adolescência é um período onde ocorrem múltiplas mudanças, que somadas aos diferentes aspectos sociais tornam o adolescente vulnerável a condições desfavoráveis à saúde podendo ocasionar diversas patologias bucais. A educação em saúde é excelente alternativa de aprendizado, delineada com objetivo de estimular ações contundentes à saúde. Deste modo, no intuito de criar um meio de educação compativel com o público adolescente, este trabalho objetivou o desenvolvimento de jogo eletrônico de estilo puzzle, como ferramenta de educação em saúde. O jogo foi desenvolvido no Laboratório de Realidade Virtual (LARV) da Universidade Federal do Pará por uma equipe multidisciplinar. Como resultados, obteve-se um jogo composto de sete fases onde o jogador tem como objetivo ganhar pontos a partir do alinhamento de peças para formação de diagnósticos, desta forma aprendendo a respeito das características de cada doença bucal, para isso um tutorial disponibiliza informações sobre sete doenças bucais. $O$ jogo foi testado pela equipe e apresenta bom funcionamento em plataformas Windows e Linux, estando apto para implantação e uso em ampla escala.

Palavras Chave: Educação, Saúde, Jogo, Adolescente.

Abstract. The adolescence is a period of multiple changes, which, summed with different social aspects, turns the adolescent vulnerable to unfavorable health conditions that can cause oral disorders. Health education is an excellent learning alternative outlined in order to encourage healthy practices. Thus, this paper aims the development of a puzzle electronic game as a tool in health education, compatible with the teen public. The game was developed by the laboratory of virtual reality (LARV) of the Federal University of Pará by a multidisciplinary team. As result, the game was concluded with seven stages in which the player has to align images to form diagnoses to get points, to guide the user the game offers a tutorial with information on seven oral diseases. The game was tested by the development team and is functioning properly on Windows and Linux platforms.

Keywords: Education, Health, Game, Teenager

\section{INTRODUÇÃO}

A adolescência é um período caracterizado por intensas mudanças biológicas, sociais e psicológicas (Ferreira, 2006). Essas alterações quando somadas a diferentes aspectos tais como: diminuição dos cuidados por parte dos pais, influência negativa das mídias e de seus grupos sociais, proporcionam condições desfavoráveis à saúde do adolescente, tornando-o vulnerável às patologias bucais (Viero,2015; Natash, 2016). A população adolescente vem demonstrando acelerado crescimento populacional, representando $29 \%$ da população mundial, sendo que $80 \%$ se encontram em países em desenvolvimento como o Brasil (Horta, 2010), que já possui cerca de 21 milhões de jovens (Unicef, 
2015). Adolescentes acumulam alto índice de doenças bucais, como doença periodontal, cárie e halitose (Viera, 2010; Fernandes, 2007; Sb Brasil, 2012), e também outras de cunho sistêmico relacionadas a comportamentos alimentares e sexuais (reflexos a saúde bucal por distúrbios alimentares, doenças sexualmente transmissíveis, uso de drogas lícitas e ilícitas e uso do piercings) (Silva,2014; Oliveira, 2013)

Para o combate das patologias bucais a educação em saúde é a estratégia mais eficaz para se intervir nesta fase, uma vez que por meio das informações e atividades de aprendizado o adolescente é conduzido a adquirir hábitos para a promoção, recuperação e manutenção da própria saúde (Souza, 2009; Sousa 2015). Os jogos eletrônicos fazem parte do cotidiano dos adolescentes e por isso muito tem se explorado sobre seu potencial como ferramenta de ensino aprendizagem (Arruda, 2011; Seixas 2014). O uso de Jogos eletrônicos no processo de educação vem se tornando tão amplo que gerou um conceito a "gameficação" que se refere à utilização de mecanismos de jogos tais como, formulação de estratégia, sistema de recompensa, regras, fases, interatividade, tentativa e diversão para potencializar o processo de ensino, a utilização destes recursos tem a finalidade de tornar os assuntos abordados nos jogos mais atrativos, promovendo desta forma o engajamento e motivação do jogador (Fardo 2013; Silva 2015; Fadel, 2014).

Adolescentes têm acesso a variadas mídias digitais, com diversas funções de comunicação e entretenimento. Esses recursos digitais podem se apresentar na forma de jogos eletrônicos. Em certos casos adolescentes chegam a gastar longos períodos jogando. No Brasil, em média, gastam cerca de duas horas e quarenta minutos diários nesta atividade (Propmark, 2016). O potencial educativo dos Jogos eletrônicos se refere a capacidade de estimular uma série de condições fundamentais para absorção de informação, tal como a atenção, envolvimento com a atividade educativa, e habilidade cognitiva. O caráter lúdico e a multiplicidade de estímulos sonoros e visuais prendem a atenção do jogador para determinado evento que está ocorrendo no jogo evitando que ele desvie sua atenção para outros elementos que possam distraí-los. (Lemos, 2015). Além disso, o empenho necessário para se alcançar determinado objetivo do jogo promove o engajamento com as tarefas propostas, conduzindo o jogador para aquisição de conhecimento (Dourado, 2015).

Além dos adolescentes estarem habituados aos jogos eletrônicos, e do fator de entretenimento que são atribuídos aos games, que facilita o processo de aprendizagem, outro ponto que favorece a utilização dos jogos como meio educativo é a possibilidade de serem utilizados de modo contínuo, uma vez que, sua acessibilidade permite que o jogador entre em contato com a informação continuamente. Comparados aos meios tradicionais de educação em saúde como as palestras educativas, observa-se que a informação chega aos adolescentes através de ações de saúde nas escolas, sendo ministradas geralmente em um período anual, desta forma a probabilidade de esquecer-se do que se foi aprendido é muito grande. De acordo com a teoria de Ebbinghaus, pesquisador pioneiro em técnicas experimentais de aprendizado, a pessoa em média esquece 75\% das informações adquiridas dentro de 48 horas (Rawi, 2015).

Nessa lógica esta pesquisa teve como objetivo desenvolver um Jogo eletrônico como forma de educação em saúde, abordando as doenças bucais mais prevalentes no período da adolescência. $O$ presente trabalho estrutura a apresentação da pesquisa da seguinte forma: Primeiramente são apresentados os métodos, ferramentas e o processo de criação aplicados no desenvolvimento do jogo eletrônico, em seguida são 
apresentados os resultados obtidos com o desenvolvimento do jogo, assim como apresentar estudos que fundamentam a importância do uso destes métodos lúdicos no processo de ensino e educação, finalmente é apresentada a conclusão do trabalho com as considerações finais dos autores.

\section{MÉTODOS}

Este trabalho trata de pesquisa laboratorial baseada no processo de potencialização de ensino e aprendizagem a partir da utilização de jogos eletrônicos. $\mathrm{O}$ jogo eletrônico de saúde bucal foi desenvolvido em parceria com o laboratório de Realidade Virtual (LARV) da Faculdade de Engenharia da Computação da Universidade Federal do Pará (UFPA). A equipe multidisciplinar composta de profissionais de odontologia, engenharia da computação, ciências da computação e artes visuais, desenvolveram um jogo de estilo puzzle baseado no alinhamento de determinadas peças para ganhar pontos.

$\mathrm{O}$ jogo foi desenvolvido em três etapas. $\mathrm{Na}$ etapa 01 foi necessário o desenvolvimento de todos os elementos ilustrativos (ícones, botões e fontes) que foram desenhados de forma artesanal, ou seja, a mão usando grafite. Na etapa 02 ocorreu processamento digital das imagens por meio do software de edição de imagens, o GIMP (Gimp, 2016), no qual as figuras foram vetorizadas, possibilitando a manipulação digital das mesmas (Fig. 01).

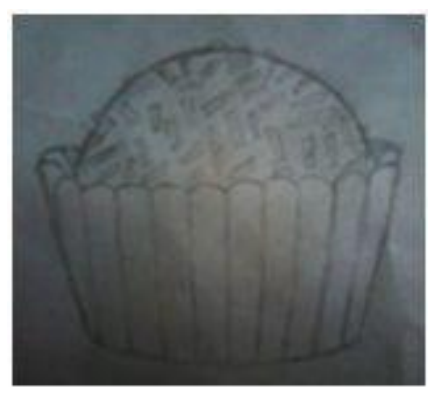

(a)

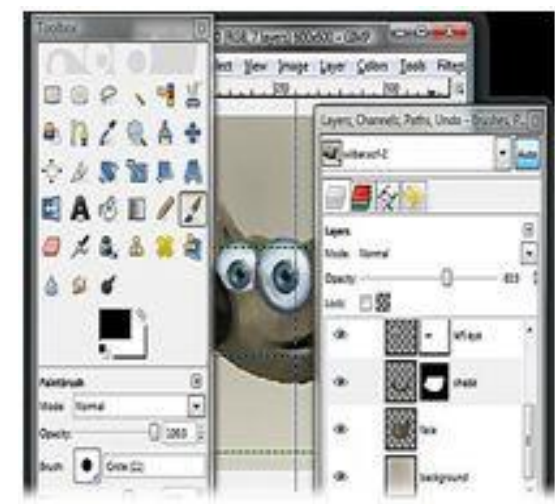

(b)

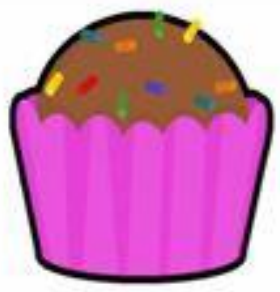

(c)

Figura 01 - (a) Desenho feito de forma artesanal; (b) Editor de imagens Gimp; (c) Imagem após passar pelo processo de edição digital. Fonte: Dados da pesquisa, LARV, 2016

Após o processamento de imagens, todos os elementos vetorizados eram transmitidos para o motor gráfico Unity3D (Unity, 2016). Ele reúne todos os elementos de um jogo como física, som e imagem e definiu a maneira de como esses elementos se relacionam definindo desta forma a dinâmica do jogo. Durante a terceira etapa o jogo foi testado pela equipe de desenvolvimento, para verificar o funcionamento correto de todas as funcionalidades inseridas, tais como: $\mathrm{O}$ reconhecimento dos cliques do mouse na tela, se os elementos que compõem a interface do jogo estavam executando suas funções corretamente e se as fases mudam de acordo com a quantidade de pontos estipulada e após esses testes o jogo foi considerado em sua versão final. 


\subsection{DESENVOLVIMENTO}

Uma vez que o desenvolvimento de um jogo exige a utilização de variadas áreas de conhecimento (Rodrigues et al. 2010), tornou-se necessário um caráter multidisciplinar por parte da equipe de desenvolvimento, que contou participação de alunos dos cursos de engenharia da computação e ciência da computação, que seriam os responsáveis pelo desenvolvimento técnico computacional propriamente dito, uma aluna do curso de odontologia que assumiu o papel de desenvolver o conteúdo teórico do game e de gerir a equipe coordenando e articulando tarefas entre os membros do grupo, tendo papel importante para direcionar a equipe quanto a assuntos específicos da área de odontologia e, por fim, a equipe também contou com uma aluna do curso de artes visuais, esta teve como função a criação de todas as imagens presentes no jogo, sendo de fundamental importância para o desenvolvimento da interface gráfica.

O processo de criação foi organizado a partir de reuniões semanais com todos os membros a equipe de desenvolvimento, nestas reuniões eram atribuídas atividades aos desenvolvedores, eram discutidas atividades pendentes de forma que cada integrante expunha suas dificuldades e a equipe às analisavam em busca de uma solução rápida e eficiente sem comprometer o desenvolvimento geral da ferramenta e por último eram repassadas atividades para cada membro. Desta forma o jogo foi desenvolvido em um período de 12 meses, onde todas as etapas de desenvolvimento do jogo foram orientadas por docentes da instituição de ensino.

\section{RESULTADOS E DISCUSSÃO}

Jean Piaget caracterizou quatro grandes períodos de desenvolvimento intelectual que dizem respeito ao que o indivíduo está apto a fazer e aprender em determinado período da sua vida, dentro desses períodos há o estágio das operações formais que ocorre entre 11 a 16 anos (Ferracioli, 1999). Segundo a teoria de Piaget nesta fase o indivíduo adquiri o ápice do padrão intelectual, ou seja, capacidade de raciocínio hipotético e dedutivo, poder de chegar a conclusões, formar esquemas conceituais, e propor novas condutas a partir do que lhe é apresentado (UNESP, 2010; Minussi 2016). Desta forma, a adolescência constitui um período chave, para inserção de práticas de educação em saúde bucal, por que esta fase ao mesmo tempo em que possui uma variedade de fatores que podem acarretar problemas bucais como consumo de álcool, drogas, obesidade, início da vida afetiva, busca por autonomia e identidade, preocupação excessiva com aparência física, dentre outros fatores (Jager, 2014), também apresenta maiores possibilidades de assimilação de informação e consequentemente implementação de práticas de autocuidado.

Entretanto, o adolescente demonstra uma falta de interesse e entusiasmo por diferentes atividades de educação, isso é resultado de alterações cerebrais que ocorrem nesta época. $\mathrm{O}$ núcleo acumbente é uma estrutura neurológica que participa do sistema de recompensa cerebral (SRC), quando fazemos algo que julgamos prazeroso como aprender algo novo, essa estrutura é ativada e recebe uma carga de dopamina gerando sensação de bem estar. Porém na adolescência ocorre a perda de até metade dos receptores dopaminérgicos do núcleo acumbente ocasionando uma falta de motivação e letargia característica entre jovens (Rossa, 2012). Essa desmotivação pode interferir no aprendizado e devido a isso o adolescente necessita de um estímulo potente para ativar o SRC, e que tenha um fator motivacional com intuito de conseguir o interesse e 
colaboração do jovem na atividade de educação em saúde.

Desenvolvido para uso nas plataformas Windows e Linux, o jogo aborda as principais doenças bucais a qual os adolescentes estão mais vulneráveis sendo elas cárie, doença periodontal, halitose, manifestações bucais de transtornos alimentares, consequências do uso do piercing bucal, câncer bucal e HPV. O jogo foi criado com intuito de solucionar o problema de ensinar um indivíduo com alta capacidade de absorção de conteúdo, mas com pouco entusiasmo para participar e interagir com a atividade educativa, já que a abordagem que geralmente é feita a esses jovens na maioria das vezes é inadequada, realizada comumente na forma de palestras semelhantes à aulas, que para maioria dos adolescentes é considerada como um método que torna difícil a compreensão dos assuntos abordados (Silva, 2015).

A escolha de usar um jogo como ferramenta de educação em saúde surgiu da análise das características que um game possui de estimular uma série de fatores essências para aquisição de conhecimento. O teor lúdico atribuído aos jogos digitais se refere ao entretenimento gerado pelo jogo promovendo o interesse e motivação pela atividade. Além disso, a possibilidade de visualização de eventos através de uma interface digital viabiliza e facilita a compreensão dos assuntos, elementos e significados que o jogo está abordando (Savi, 2008). O jogo desenvolvido neste trabalho propôs uma inovação na prática de educação em saúde a fim de envolver o adolescente e motivá-lo a aprender abordando assuntos de saúde bucal que podem ser desenvolvidos ao longo da adolescência sobre a influência de diferentes fatores.

No Jogo de saúde bucal nomeado de katzuru, que na língua indígena "Tenetehara" quer dizer boca saudável, inicialmente há a tela inicial onde o jogador é conduzido às instruções que exemplificam como o jogo funciona, e depois oferece um tutorial sobre as doenças que são abordadas com informações de sua causa, fator desencadeante e consequência clínica (Fig. 02). Essas informações permitirão a interpretação dos ícones que serão dispostos no grid, para que o jogador associe as figuras e alinhe-as para formar um diagnóstico e consequentemente ganhar pontos.

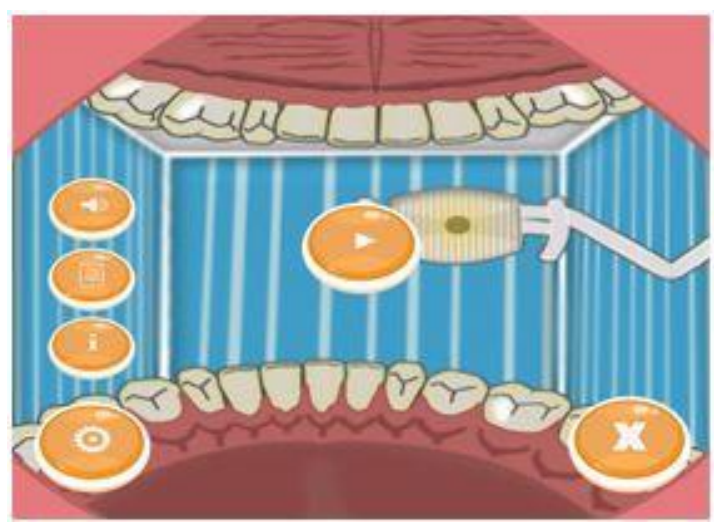

(a)

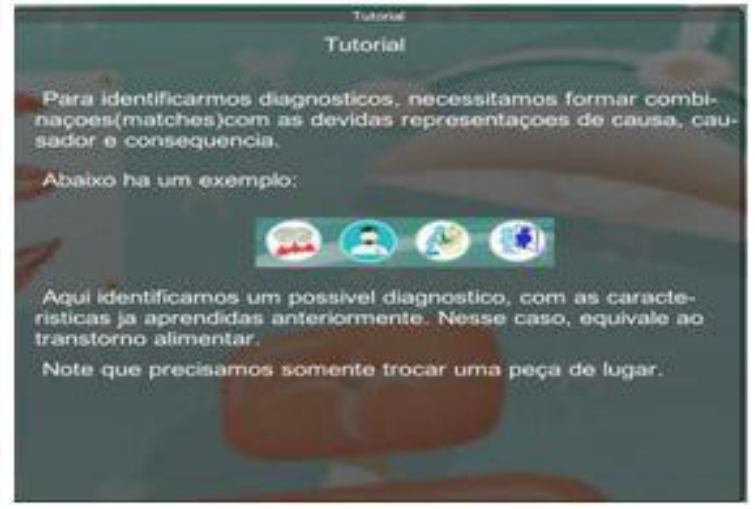

(b)

Figura 02 - (a) Tela inicial do jogo; (b) Tutorial exemplificando como ocorre a formação das combinações entre as figuras. Fonte: Dados da pesquisa, LARV 2016.

Como a dinâmica do jogo baseia-se no alinhamento de ícones que representam a causa, fator desencadeante e consequência de cada doença para formação de diagnósticos, é necessário que o jogador interprete o significado de cada ícone. Por esse 
motivo ele é instruído a ir para o tutorial (Fig. 03), onde há informações de cada doença. É nesta parte do jogo que ele então tem acesso à descrição da doença mostrando quais fatores podem desencadeá-las e qual sua consequência clínica, possibilitando que o jogador interprete o significado de cada ícone e consequentemente associe os ícones que estão relacionados. Desta forma, ao ler as informações, tentar associar com a figura, interpretar seu significado e relacionar com os ícones correspondentes, o adolescente não está apenas adquirindo conhecimento sobre as doenças, mas construindo um saber mais aprofundado, pois, diferentemente das palestras onde ele apenas recebe as informações, no jogo, ele recebe processa e reflete sobre as informações para poder aplicá-las no game, tornando o aprendizado ativo.

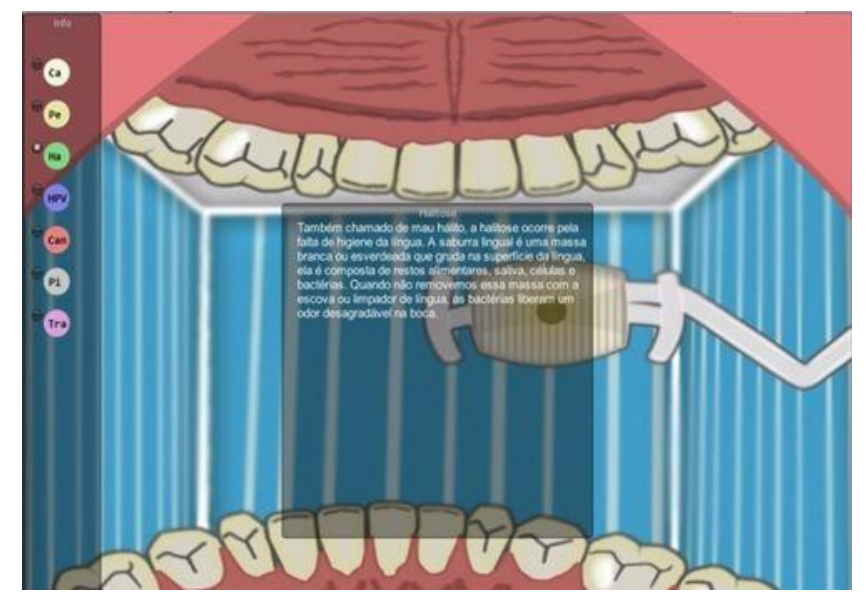

Figura 03 - Descrição das características das doenças. Fonte: Dados da pesquisa LARV 2016

No jogo de saúde bucal, as peças que o jogador deve alinhar são chamadas de ícones, onde estes estão dispostos em um "grid", que pode ser interpretado como uma matriz que guarda em cada célula um ícone e cada um é referente às características clínicas de cada doença, que quando alinhadas, da maneira correta, formam um diagnóstico. Cada diagnóstico possui três ícones cada um deles se refere à causa, fator desencadeante e consequência clínica de cada doença.

O jogo é dividido em sete fases, apresentando variadas peças geradas aleatoriamente, essas se destroem quando combinadas recompensas correspondentes que compõem um determinado diagnóstico, e logo após outras peças surgem, mantendo sempre o grid de ícones completo. O jogador terá um determinado tempo em cada fase, caso o tempo expire ele voltará para fase inicial até que consiga formar um número de diagnósticos em determinado tempo. (Fig. 4a).

Para motivar o jogador, o jogo possui um "mapa" (Fig. 4b) que se apresenta como uma boca com algumas doenças e ao decorrer do jogo, na medida que o jogador ganha pontos, essas doenças vão desaparecendo. 


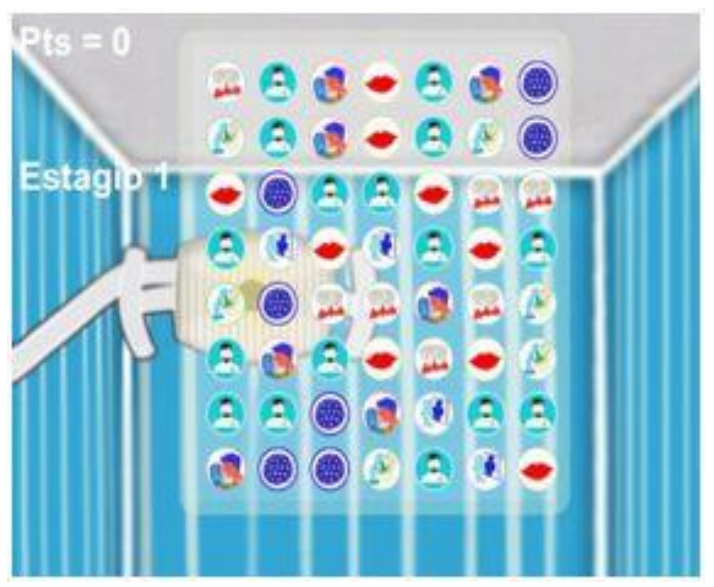

(a)

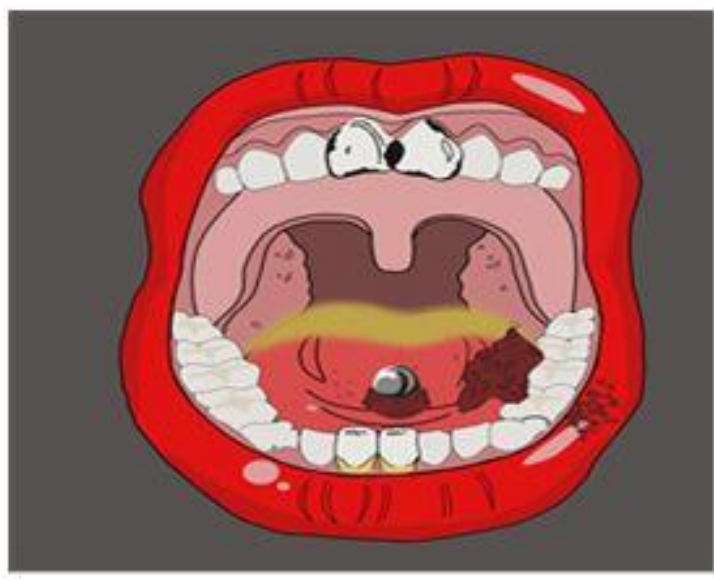

(b)

Figura 04 - (a) Grid de peças; (b) Mapa das doenças no jogo Fonte: Dados da pesquisa, LARV 2016

As doenças que o jogador não conseguir aprender, ou seja, não conseguir formar os diagnósticos, continuarão aparecendo nas fases seguintes até que o jogador consiga fazer as combinações das figuras para formar o diagnóstico desta doença.

Em cada fase existem peças coringa representada pela figura do dentista, esta figura aparece como bônus quando o jogador atinge um determinado número de pontos. Caso o jogador consiga alinhar três peças coringas, ela destrói uma coluna inteira de peças na horizontal ou vertical.

O jogo é do estilo puzzle (quebra-cabeça), que se caracteriza por possuir um número grande de elementos que entram e saem do campo visual do jogador (Oey, 2014). Isto estimula o aumento da atenção e o foco, acionando o que chamamos de atenção seletiva, que ocorre quando é necessário adquirir informações para o cumprimento de determinadas tarefas em um curto espaço de tempo (Ferreira, 2016). No jogo, para formação do diagnóstico, o jogador terá que alcançar um determinado número de pontos em um determinado intervalo de tempo, para isso, terá que ficar atento aos ícones, planejar sua movimentação, e ao mesmo tempo interpretar seus significados baseados nas informações aprendidas no tutorial. Esse grande gerenciamento de funções poderá fazer com que ele aumente sua atenção para as informações e eventos do jogo, contribuindo desta forma para a fixação das características das doenças. Além disso, espera-se que a motivação gerada para alcançar os pontos e consequentemente evoluir de fase, fará com que o jogador várias vezes volte ao tutorial para gravar as características das doenças, até o ponto em que estas já ficaram retidas na memória.

\section{CONCLUSÃO}

Deste modo, considera-se o jogo eletrônico como excelente opção de educação em saúde para adolescentes, pois além de fazer parte do contexto em que estes estão inseridos, possuem características essências para a aquisição de conhecimento. $\mathrm{O}$ jogo constitui potente alternativa como meio auxiliar e adicional as práticas de educação em saúde bucal e está apto para iniciar a fase experimental de campo, apresentando 
estrutura delineada para o adolescente não só aprender sobre as doenças bucais a qual está mais vulnerável, mas também identificar os fatores a que se expõem mediante escolhas e comportamentos, alcançando, desta forma, os objetivos propostos pela educação em saúde.

\section{REFERÊNCIAS}

ARRUDA E. P. O papel dos videogames na aprendizagem do conceito e analogias históricas pelos jovens. Ensino Em Re-Vista, v.18, n.2, p.287-297, dez. 2011

DOURADO J. B; SANTOS A. B; SILVA J. S; SILVA F. J. M; BORTOLli B; BEZERRA A; Desenvolvimento e Avaliação de um jogo de tecnologia de RA para auxiliar no ensino da matemática In: Simpósio Brasileiro de Games 2015, Teresina. Anais. Teresina: Faculdade de Tecnologia de Lins Professor Antônio Seabra (FATEC) 2015, p. 846-853.

FADEL L. M; ULBRICHT V. R; BATISTA C. R; VANZIN T. Gameficação na educação. São Paulo: Pimenta Cultural. 2014 300p.

FARDO M. L. A Gameficação Aplicada em Ambientes de Aprendizagem. Revista Novas Tecnologias na Educação v.11 n.1 p. 02-09 jul 2013

FERNANDES C. B; AQUINO D. R; FILHO J.C; CORTELlI S. C; CORTELLI J.R. Prevalência de microrganismos periodontais intra e extra sulcular em crianças adolescentes e adultos jovens. Revista Ciências odontológicas Brás v.10 n.3 p.90-97. 2007.

FERRACIOLI, L. Aprendizagem desenvolvimento e conhecimento na obra de Jean Piaget: Uma analise do processo de ensino-aprendizado em ciências. Revista Brasileira de Estudos Pedagógicos v.80 n.194 p.05-18. 1999

FERREIRA C. P. S et al. Estratégias pedagógicas para educação em saúde com adolescentes: uma revisão integrativa Revista de Pesquisa Cuidado é Fundamental. v.8 n.2 p 4197-4211 2016.

FERREIRA M. A. A educação em saúde na adolescência: Grupos de discussão como estratégia de pesquisa e cuidado educação. Revista Texto Contexto Enfermagem v.15 n.2 p.205-211. 2006

GIMP The GNU ImageManipulationProgram Software de edição de imagem Disponível em:<http//: www. Gimp> Acesso em 28 ago 2016.

HORTA N.C; SENA R.R. Abordagem ao adolescente e ao jovem nas políticas públicas de saúde no Brasil: um estudo de revisão.Physis Revista de saúde coletiva, Rio de janeiro, v.20 n.2 p.475- 495, 2010.

JAGER E.M; BATISTA F. A; PERRONE M. C; SANTOS S.S; DIAS A. C. G; O 
adolescente no contexto da saúde pública brasileira reflexões sobre o PROSAD. Revista Psicologia em Estudo, v.19 n. 2 p. 211 -221Jun 2014.

LEMOS. L, Games na promoção e educação em saúde: práticas de significação In: IV Colóquio Semiótico das Mídias 2015, Alagoas. Anais. Alagoas. Centro Internacional de Semiótica e Comunicação. 2015 p. 01-15

MINUSSI, M.M. SOUZA A.T. Web-Game educacional para ensino e aprendizagem de ciências. Revista Novas Tecnologias na Educação. Rio Grande do Sul,v.14, n.2, julho 2016.

NATASH Y; SANTOS D.S; LEITE I.M.L. Saúde e Doença: Percepção de adolescentes que vivenciaram o lúdico como estratégia de educação em saúde.Revista de enfermagem da UFPE,Recife n.5 v.10 p.1822-1827. 2016.

OEI C. A; PATTERSON M.D. Playing a puzzle video game with changing requirements improves executive functions Revista Computers in Human Behavior v.37 p 216-228 Ago. 2014.

OLIVEIRA D.C; PEREIRA P.N; FERREIRA F.M; PAIVA S.M; FRAIZ F.C; Impacto relatado das alterações bucais na qualidade de vida de adolescentes: Revisão Sistemática. Pesquisa Brasileira em Odontopediatria e Clínica integrada. João Pessoa v.13 n.1, p 123-129 mar. 2013

PROPMARK Publicado por João Coscelli aborda o uso de smartphones como meios para jogar. Disponível em <http://propmark.com.br/mercado/games-ganham-espaco-entre-smartphones> acessado em 24 abr. 2016.

RAWI A. W Development of a mobile device optimized cross plataforma oral pathology and radiology spaced repetition system for dental education. Revista Journal of Dental education. v.79 n.4 p 439-447 abril 2015.

RODRIGUES, H. F.; MACHADO, L. S. E.; VALENÇA, A. M. G.; Definição e Aplicação de um Modelo de Processo para o Desenvolvimento de Serious Games na Área de Saúde. Proc. Congresso da Sociedade Brasileira de Computação - Workshop de Informática Médica, p. 1532-1541, 2010.

ROSSA A. A. O. O Sistema de Recompensa do Cérebro Humano. Revista. Textual. v.16 n.02 p.04-11 out. 2012.

SAVI R; ULBRICHIT V R; Jogos digitais educacionais: Benefícios e Desafios. Revista Novas Tecnologias na Educação.v.6 n 02 dez. 2008.

SB BRASIL 2010 Pesquisa Nacional de Saúde Bucal. Desenvolvido pelo Ministério da Saúde Brasília 2012. Apresenta os principais resultados da pesquisa de saúde bucal. 
Disponível

em:

http://docplayer.com.br/1668608-Ministerio-da-saude-pesquisa-nacional-de-saude-buca l-sb-brasil-2010-resultados-principais.htmlAcesso em 14 de abril de 2016.

SEIXAS L. R; GOMES A. S; FILHO I. J. M; RODRIGUES R. L; Gameficação como estratégia no engajamento de estudantes no ensino fundamental In: XXV Simpósio brasileiro de informática na educação, 2014 Pernambuco. Anais Pernambuco:Grupo de Pesquisa Ciências Cognitivas e Tecnologia Educacional/Universidade Federal de Pernambuco, 2014 p 559-568.

SILVA P. A; MARTINS V. F; DUTRA C; MACHADO T. L. A; ARAÚJO L.F. A.Gamificação para melhoria do engajamento do ensino médio integrado In: XIV Simpósio Brasileiro de Games 2015. Anais Teresina: Centro de Estudos em Sistemas Avançados do Recife/ Escola Técnica Estadual Cícero Dias, NAVE, Brasil 2015 p. 794-801.

SILVA, Raimunda Magalhães; CATRIB Ana Maria Fontenelle. Promoção de saúde na adolescência e Concepções de Cuidados $1^{\circ}$ Edição. Fortaleza: CEEdUECE, 2014.j $454 \mathrm{p}$.

SOUZA I. P. M. A; JACOBINA R. R. Educação em saúde e suas versões na história brasileira. Revista Baiana de Saúde Pública. v.33 n..4 p 618-627.dez. 2009.

SOUSA .P; GASPAR P, FONSECA H, HENDRICKS C, MURDAUGH C. Health promoting behaviors in adolescence: validation of the Portuguese version of the adolescent life style profile Revista J. Pediatr v.91 n.4 p 358-365, agosto 2015.

UNICEF BRASIL Fundo das Nações Unidas Para Infância. Relatório da situação da adolescência brasileira Disponível em: http://www.unicef.org/brazil/pt/media_22244.htmacesso em 29 de mar. 2015

UNITY3D. Unity - Overview. Software para desenvolvimento de Jogos. Disponível em: $<$ https://unity3d.com/pt/unity $>$. Acesso em 28 de ago 2016.

UNIVERSIDADE ESTADUAL PAULISTA: Caderno de formação de professores educação infantil: princípios e fundamentos. São Paulo, 2010. 01p (UNESP-Vol1)

VIERA T.R; PERÉT A.C.A; PERET L.A. Alterações Periodontais Associadas às Doenças Sistêmicas Entre Crianças e Adolescentes. Revista Paulista de Pediatria v.28 n. 2 p. $237-243.2010$

VIERO V.S.F; FARIAS J.M; FERRAZ F; SIMÕES P.W; MARTINS J.B; CERETTA L.B. Educação em saúde com adolescentes: análise da aquisição de conhecimento sobre temas da saúde. Esc. Anna Nery Revista de Enfermagem v.19 n.3 p.484-490. 2015. 